\title{
Condições de drenagem relacionadas ao trânsito de máquinas em solo de várzea (RS-Brasil)
}

\author{
José A. Louzada ${ }^{1}$, Nelson Caicedo ${ }^{1}$ \& Fernanda Helfer ${ }^{1}$
}

\begin{abstract}
RESUMO
A exploração dos solos de várzeas do Rio Grande do Sul, Brasil, tem sido historicamente restrita ao binômio arroz irrigado-pecuária extensiva. Dentre os diversos fatores que contribuem para este quadro, as más condições de drenagem são dos mais restritivos, haja vista que, especificamente em relação ao arroz irrigado, tal situação acarreta atraso no plantio com conseqüente queda da produtividade. Como objetivo inicial, o modelo SWAP foi validado com dados de um solo típico das várzeas do Rio Grande do Sul, para posteriormente se empregá-lo na simulação de diversos cenários sob diferentes condições de drenagem e os resultados dessas simulações foram confrontados com critérios que avaliam a possibilidade de preparo do solo, com base em sua umidade. Este confronto mostrou haver relação muito estreita entre a drenagem subsuperficial e superficial; quando esta é satisfatória, os perfis de umidade podem ser compatíveis com o preparo do solo, mesmo com drenos subsuperficiais bastante espaçados; caso contrário, o crescimento no número de dias aptos para a mecanização exige a presença de drenos subsuperficiais muito próximos.
\end{abstract}

Palavras-chave: simulação, manejo do solo, arroz irrigado

\section{Drainage conditions related with mechanization in flood plain soil (RS-Brazil)}

\begin{abstract}
The exploration of flood plains soils in the State of Rio Grande do Sul (Brazil) has been historically restricted to the binomial 'irrigated rice crop - livestock'. Among several factors that contribute to that situation, the bad drainage conditions are one of the most important. In relation to irrigated rice, this situation promotes late planting and, consequently, productivity reduction. After validation with measured data in a typical flood plain soil of Rio Grande do Sul, the SWAP model was used to simulate scenarios under different drainage conditions. The results were compared with criteria that evaluate soil tillage possibility based on moisture values. This comparison showed a close relationship between subsurface and surface drainage. When surface drainage is satisfactory, soil moisture contents can be compatible with soil tillage, even if subsurface drains are too widely spaced. Otherwise, the increase in number of workable days for mechanization requires very closely-spaced subsurface drains.
\end{abstract}

Key words: simulation, soil tillage, irrigated rice crop 


\section{INTRODUÇÃO}

Os solos de várzea do Rio Grande do Sul incorporados ao processo produtivo, totalizam uma área ao redor de 3.000.000 ha; seu modelo de exploração está assentado na pecuária extensiva e, fundamentalmente, no arroz irrigado. Com rendimento médio próximo de $6.000 \mathrm{~kg} \mathrm{ha}^{-1}$ contra cerca de $2.000 \mathrm{~kg} \mathrm{ha}^{-1}$ do restante do País, a produção de arroz do Rio Grande do Sul representa mais de $40 \%$ da produção nacional devido, em grande parte, ao fato de que, nesse Estado, predomina a lavoura irrigada enquanto na maioria dos demais estados produtores também se encontram lavouras de sequeiro. Na composição percentual do Valor Bruto da Produção (VBP) da agropecuária gaúcha, o arroz lidera o 'ranking', tendo atingido $17 \%$ de participação no ano de 1995; este valor chegou a 25,63\% em 1991 (Grando, 1996); ao final da década de 90, a orizicultura gaúcha chegou muito próxima de $50 \%$ da produção nacional de arroz, contribuindo com 23\% do Produto Interno Bruto do Estado (Agrianual, 1999). O arroz representa 33,5\% da produção dos cultivos de verão no Rio Grande do Sul, superando a soja, que engloba 32\%. Cerca de $85 \%$ da colheita gaúcha são exportados para outros estados, o que solidifica a condição do Rio Grande do Sul, de Estado fornecedor de alimentos de consumo básico da população brasileira. No contexto internacional em que o Brasil, juntamente com o Japão, é o décimo maior produtor, a produtividade gaúcha se equipara à de alguns dos maiores produtores mundiais (Grando, 1996).

Apesar dos dados anteriormente apresentados constituírem um quadro aceitável para o Rio Grande do Sul, o modelo de exploração restrito ao arroz irrigado e à pecuária apresenta rendimentos inferiores ao potencial existente; além de questões econômicas e de estrutura fundiária, tratadas politicamente de forma precária, também os solos e o clima apresentam barreiras naturais à utilização de um modelo mais adequado; no caso da pecuária, a implantação de pastagens cultivadas que possibilitem uma utilização intensiva, é dependente de melhores condições de drenagem e, eventualmente, também de irrigação suplementar e, em se tratando da agricultura, o mesmo pode ser dito quando se busca viabilizar a introdução de culturas alternativas ao arroz; no caso específico do arroz irrigado, há problemas de drenagem nos períodos de preparo do solo e colheita e alta demanda de água para irrigação; quando do preparo, as precipitações, associadas às más condições naturais de drenagem, têm retardado o plantio em aproximadamente $50 \%$ dos anos, como conseqüência, em cada um desses anos ocorre, em média, uma queda de produtividade ao redor de $20 \%$; esses são valores que representam observações feitas pelo Instituto Riograndense do Arroz (IRGA) ao longo de vários anos. A colheita ocorre com o solo saturado, o que acarreta sua desestruturação e exige a presença de máquinas que representam um alto custo para o agricultor. Este conjunto de informações evidencia que um aperfeiçoamento no modelo de exploração desses solos passa, necessariamente, por melhores condições de drenagem, razão por que se trata aqui exclusivamente das relações da drenagem com o trânsito de máquinas, com o objetivo de: i) validar o modelo SWAP (van Dam et al.,1997; van Dam, 2000); ii) apresentar os resultados de suas simulações em cenários que diferem pelas condições de drenagem e iii) confrontar esses resultados com critérios que definem condições de umidade adequadas para o preparo do solo.

\section{MATERIAL E MÉTODOS}

\section{Área experimental}

A parcela experimental, com área de 1,0 ha, conta com um sistema de drenagem subsuperficial e superfície sistematizada. Há um único plano com declividade de 0,15\% que faz com que a água superficial escoe na direção do coletor. O sistema subsuperficial é composto por tubos de PVC, não envelopados, com $35 \mathrm{~m}$ de comprimento instalados com a mesma declividade da superfície. O solo é típico da várzea arrozeira do Rio Grande do Sul e ocorre no complexo da Estação Experimental do Arroz (EEA) do IRGA, região metropolitana de Porto Alegre, capital do Rio Grande do Sul; sua classificação taxonômica é Planossolo Hidromórfico eutrófico arênico (EMBRAPA, 1999) e seus parâmetros físicos são apresentados na Tabela 1.

Tabela 1. Caracterização física do solo da área experimental

\begin{tabular}{lc}
\hline Característica & Valor \\
Porosidade (\%) & 39,0 \\
Condutividade hidráulica saturada de A $\left(\mathrm{m} \mathrm{d}^{-1}\right)^{\star * \star *}$ & 0,153 \\
Espessura do horizonte A $(\mathrm{m})$ & 0,75 \\
Condutaividade hidráulica saturada de B $\left(\mathrm{m} \mathrm{d}^{-1}\right)$ & Impermeável $^{*}$ \\
Espessura do horizonte B (m) & $>2$ \\
\hline Curva de retenção [h $\left.(\mathrm{cm})^{\star} \theta\left(\mathrm{cm}^{3} \mathrm{~cm}^{-3}\right)\right]$ & 0,38 \\
60 & 0,36 \\
100 & 0,34 \\
200 & 0,33 \\
300 & 0,31 \\
500 & 0,29 \\
700 & 0,26 \\
1000 & 0,25 \\
2000 & 0,24 \\
3000 & \\
$\mathrm{~m}^{*}(-)$ & 0,05049 \\
$\alpha_{\mathrm{d}}{ }^{* * *}\left(\mathrm{~cm}^{-1}\right)$ & 1,1794 \\
$\theta_{\mathrm{r}}\left(\mathrm{cm}{ }^{3} \mathrm{~cm}^{-3}\right)$ & 0,01326 \\
$\mathrm{n}(-)$ & 0,02652 \\
\hline Parâmetros da equação de van Genuchten & 0,1521 \\
\hline
\end{tabular}

"Não ocorreu fluxo através da amostra após $72 \mathrm{~h}$ no permeâmetro; ** Parâmetro $\alpha$ da curva de drenagem; ${ }^{* * *}$ Parâmetro $\alpha$ da curva de umedecimento; ${ }^{* * * *}$ Método do poço na presença de lençol freático

Apresentam-se na Figura 1 em planta e em corte, as principais características do sistema de drenagem existente na área experimental.

\section{Dados climáticos}

Obtiveram-se os dados climáticos diários, precipitação e evaporação de cada período simulado em uma estação 
A.

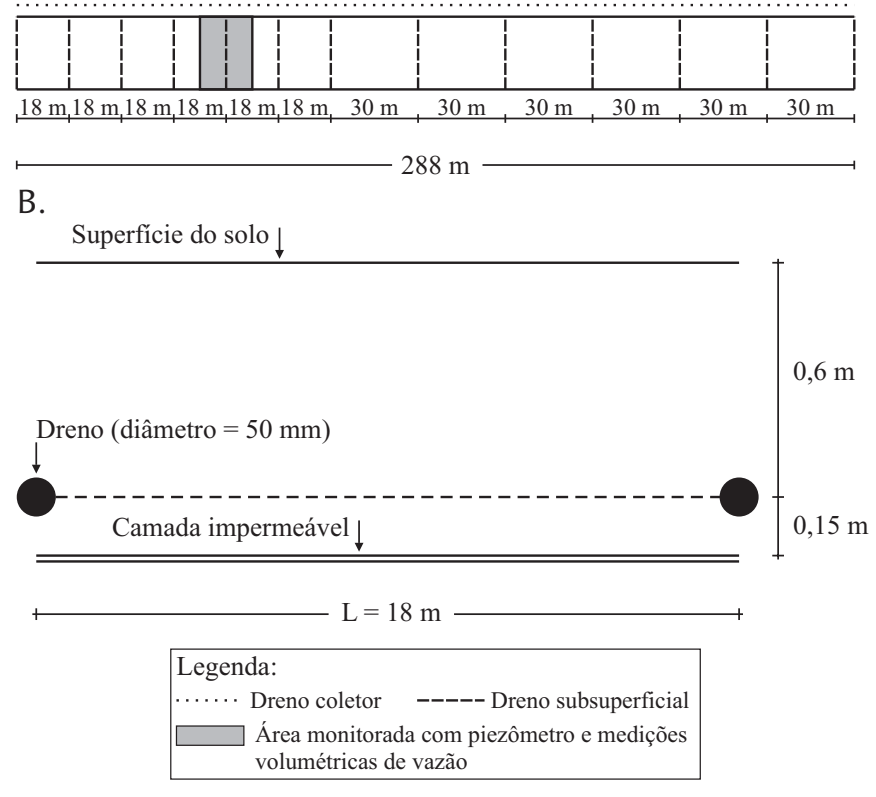

Figura 1. (A) Planta baixa da área experimental de drenagem e (B) Corte transversal junto à área monitorada

meteorológica existente junto à área experimental, na qual os registros de precipitação são feitos em pluviômetro e os de evaporação em Tanque Classe A.

\section{O modelo SWAP \\ Equações fundamentais}

O embasamento físico do modelo SWAP (van Dam et al., 1997; van Dam, 2000) é a equação de Richards, que resulta da combinação das equações de continuidade e de Darcy; em sua versão mista, utilizada no modelo, a equação de Richards tem a forma que segue.

$$
\frac{\partial \theta}{\partial t}=\frac{\partial\left[K(h)\left(\frac{\partial h}{\partial z}+1\right)\right]}{\partial z}-W(h)
$$

em que:

$$
\text { h - potencial de pressão, } \mathrm{L}
$$

$\mathrm{K}(\mathrm{h})$ - condutividade hidráulica, $\mathrm{L} \mathrm{T}^{-1}$

$\theta$ - umidade volumétrica, $\mathrm{L}^{3} \mathrm{~L}^{-3}$

$\mathrm{t}$ - tempo, $\mathrm{T}$

z - coordenada vertical (+ para cima), L

W(h) - composição de variáveis que representam entradas (+) ou saídas (-), T-1

Esta forma da equação garante a correção no balanço de massa e a continuidade da solução em meios heterogêneos, ou seja, agrega as vantagens das formas baseadas isoladamente na variável $\theta$ e na variável h (Celia et al., 1990). Nesta aplicação do modelo, onde não há cultivo, o termo W(h) representa uma saída através do sistema de drenagem subsuperficial.

Tendo em vista sua natureza hiperbólica e acentuada nãolinearidade, a equação de Richards só pode ser resolvida numericamente; o modelo emprega um esquema dito implícito com linearização explícita, ou seja, as derivadas são estimadas no tempo $(\mathrm{t}+1)$ e as relações $\mathrm{K}(\mathrm{h})$ e $\mathrm{W}(\mathrm{h})$ no tempo (t). A equação discretizada é apresentada a seguir, donde $\mathrm{h}_{\mathrm{i}}{ }^{\mathrm{j}+1, \mathrm{p}}$ é a única incógnita.

$$
\begin{aligned}
& \mathrm{C}_{\mathrm{i}}^{\mathrm{j}+1, p-1}\left(\mathrm{~h}_{\mathrm{i}}^{\mathrm{j}+1, \mathrm{p}}-\mathrm{h}_{\mathrm{i}}^{\mathrm{j}+\mathrm{p}-\mathrm{p}}\right)+\left(\theta_{\mathrm{i}}^{\mathrm{j}+\mathrm{p}-1}-\theta_{\mathrm{i}}^{\mathrm{j}}\right)= \\
& \frac{\Delta \mathrm{t}^{\mathrm{j}}}{\Delta \mathrm{z}_{\mathrm{i}}}\left[\mathrm{K}_{\mathrm{i}-1 / 2}^{\mathrm{j}}\left(\frac{\mathrm{h}_{\mathrm{i}-1}^{\mathrm{j}+1, \mathrm{p}}-\mathrm{h}_{\mathrm{i}}^{\mathrm{j}+1, \mathrm{p}}}{\Delta \mathrm{z}_{\mathrm{u}}}\right)+\mathrm{K}_{\mathrm{i}-1 / 2}^{\mathrm{j}}-\mathrm{K}_{\mathrm{i}+1 / 2}^{\mathrm{j}}\left(\frac{\mathrm{h}_{\mathrm{i}}^{\mathrm{j}+1, \mathrm{p}}-\mathrm{h}_{\mathrm{i}+1}^{\mathrm{j}+1, \mathrm{p}}}{\Delta \mathrm{z}_{\mathrm{l}}}\right)-\mathrm{K}_{\mathrm{i}+1 / 2}^{\mathrm{j}}\right]-\Delta \mathrm{t}^{\mathrm{j}} \mathrm{W}_{\mathrm{i}}^{\mathrm{j}}
\end{aligned}
$$

em que:

$$
\begin{aligned}
\mathrm{C}_{\mathrm{i}}^{\mathrm{j}+1, \mathrm{p}-1}= & {\left[\frac{\mathrm{d} \theta}{\mathrm{dh}}\right]_{\mathrm{j}+1, \mathrm{p}-1}, \mathrm{~L}^{-1} } \\
\Delta \mathrm{t}_{\mathrm{j}} & - \text { intervalo de tempo, } \mathrm{T} \\
\Delta \mathrm{z}_{\mathrm{i}} & - \text { comprimento do compartimento do perfil cor- } \\
& \text { respondente ao nó i, } \mathrm{L} \\
\Delta \mathrm{z}_{\mathrm{u}} & - \text { distância entre os nós i e } \mathrm{i}-1, \mathrm{~L} \\
\Delta \mathrm{z}_{\mathrm{l}} & - \text { distância entre os nós i e } \mathrm{i}+1, \mathrm{~L}
\end{aligned}
$$

Sua aplicação a cada nó da malha numérica $(\Delta \mathrm{x}-\Delta \mathrm{t})$ resulta em uma matriz do tipo tri-diagonal, cuja solução pode ser obtida de forma bastante eficiente. Os símbolos i, j e p se referem respectivamente ao espaço, ao tempo e às iterações, enquanto o valor médio de $K$ entre nós (i-1/2 e i+1/2) é estimado pela média aritmética dos valores de $\mathrm{K}$ nos nós i-1,i e i,i+1 e o intervalo de tempo é otimizado durante a simulação, de acordo com o número de iterações necessárias para que ocorra a convergência, sendo os intervalos máximo e mínimo previamente definidos. O critério de convergência se baseia nas variáveis $\theta$ (nó situado em compartimento não saturado) e h (nó situado em compartimento saturado). Como a derivada de $\theta$ em relação a h é nula em meio saturado, a discretização posiciona o termo que a representa $(\mathrm{C})$ unicamente no numerador garantindo, assim, a aplicação da equação nas zonas não-saturada e saturada.

\section{Discretização do perfil, condições iniciais e de contorno}

A discretização do perfil (horizonte A com espessura de $75 \mathrm{~cm}$ ) foi estabelecido em 18 compartimentos, sendo de $1 \mathrm{~cm}$ a espessura dos 10 primeiros (de 1 a 10), de $5 \mathrm{~cm}$ os próximos três (de 11 a 13) e de $10 \mathrm{~cm}$ os demais (de 14 a 18).

Como condição de contorno inferior (base do compartimento 18) adotou-se "fluxo zero", o que se justifica pelas características do horizonte B. As condições de contorno na superfície são continuamente atualizadas pelo modelo dependendo do que prevalece em cada iteração, infiltração ou evaporação. As condições iniciais para cada simulação foram definidas com base na precipitação dos cinco dias antecedentes.

\section{Evaporação}

Todas as simulações foram conduzidas sem presença de cultura. Os valores máximos de evaporação do solo desnudo $\left(E_{m}\right)$ foram admitidos iguais a 50\% dos valores registrados no tanque, ou seja, adotou-se um coeficiente de transformação igual a 0,5 (Matzenauer et al., 2002). Os valores reais de evaporação $\left(E_{r}\right)$ são definidos pelo modelo, com base na equação de Darcy, a qual é aplicada entre o primeiro compartimento e a superfície, e seu resultado $\left(\mathrm{E}_{\mathrm{D}}\right)$ confrontado com $E_{m}$. Se $E_{D} \geq E_{m}$, então $E_{r}=E_{m}$; caso contrário, $E_{r}=E_{D}$. 
Relações umidade $(\theta)$ - condutividade hidráulica $(\mathrm{K})$ potencial matricial $(\mathrm{h})$

Os valores de $\theta$, K e h são relacionados pelo modelo de van Genuchten-Mualem (Mualem, 1976; van Genuchten, 1980). Os parâmetros desse modelo já foram apresentados, quando da caracterização do solo. A histerese é considerada através dos conceitos propostos por Kool \& Parker (1987), nos quais somente as curvas principais de secagem e molhamento devem ser previamente conhecidas. Admite-se que $\theta_{\text {sat }}$ e $\theta_{\text {res }}$ são iguais para as duas curvas e a relação $K(\theta)$ é única; para tanto, também se tem, necessariamente, os valores de $\mathrm{m}$ e $\mathrm{n}$ iguais para as duas curvas principais; assim sendo, essas curvas diferem unicamente pelo parâmetro $\alpha$ e, para definição de $\alpha_{w}$, admitiu-se uma relação empírica que propõe $\alpha_{\mathrm{w}}=2 \alpha_{\mathrm{d}}$ (Kroes et al., 1998).

\section{Drenagem superficial}

As condições de drenagem superficial são consideradas, pelo modelo, com base em um parâmetro definido em um arquivo de entrada; este parâmetro estabelece a lâmina que antecede o início do escoamento superficial (ponding) e, sempre que ocorrer uma lâmina excedente, ela será admitida como instantaneamente drenada.

\section{Drenagem subsuperficial}

O fluxo horizontal a drenos paralelos instalados na mesma profundidade, é estimado com o emprego da equação de Hooghoudt e, quando aplicada a perfis homogêneos repousando sobre camada impermeável, esta equação tem a forma que segue.

$$
\mathrm{L}^{2}=\frac{8 \mathrm{~K}_{\mathrm{sat}} \mathrm{dh}_{\mathrm{s}}+4 \mathrm{~K}_{\mathrm{sat}} \mathrm{h}_{\mathrm{s}}^{2}}{\mathrm{q}}
$$

em que:

$\mathrm{L}$ - espaçamento entre drenos, $\mathrm{L}$

$\mathrm{K}_{\mathrm{sat}}$ - condutividade hidráulica saturada, $\mathrm{L} \mathrm{T}^{-1}$

$\mathrm{d}$ - espessura equivalente, $\mathrm{L}$

$\mathrm{h}_{\mathrm{s}}$ - posição do lençol freático no ponto médio entre os drenos, L

$\mathrm{q}$ - vazão específica dos drenos, $\mathrm{L} \mathrm{T}^{-1}$

A variável d permite que se incorpore o fluxo radial à equação de Hooghoudt, já que esta, originalmente, só considera o fluxo horizontal; a estimativa de d é feita com as equações que seguem (van der Molen \& Wesseling, 1991).

$$
\begin{gathered}
\mathrm{d}=\frac{\pi \mathrm{L}}{8 \ln \frac{\mathrm{L}}{\mathrm{u}}+8 \mathrm{~F}(\mathrm{x})} \\
\mathrm{F}(\mathrm{x})=\sum_{\mathrm{n}=1}^{\infty} \frac{4 \exp (-2 \mathrm{nx})}{\mathrm{n}[1-\exp (-2 \mathrm{nx})]} ; \mathrm{n}=1,3,5 \ldots
\end{gathered}
$$

A Eq. 5 apresenta uma convergência rápida para $\mathrm{x}>0,5$ sendo, neste caso, usada em combinação com a Eq. 4 para estimar a espessura equivalente; o valor de x é dado pela equação abaixo.

$$
\mathrm{x}=\frac{2 \pi \mathrm{D}}{\mathrm{L}}
$$

Para $\mathrm{x} \leq 0.5$ a convergência da Eq. 5 é muito lenta, caso em que a Eq. 4 é utilizada juntamente com a que segue.

$$
\mathrm{F}(\mathrm{x})=\frac{\pi^{2}}{4 \mathrm{x}}+\ln \frac{\mathrm{x}}{2 \pi}
$$

em que:

$\mathrm{d}$ - espessura equivalente, $\mathrm{L}$

$\mathrm{L}$ - espaçamento entre drenos, $\mathrm{L}$

u - perímetro molhado do dreno, $\mathrm{L}$

D - distância dos drenos à camada impermeável, L

Para definir o termo correspondente à drenagem subsuperficial na equação de Richards, a equação de Hooghoudt assume a seguinte forma.

$$
\eta=\frac{h_{s}}{q}=\frac{L^{2}}{8 K_{s a t} d+4 K_{s a t} h_{s}}
$$

A variável $\eta$ tem dimensão de tempo e é interpretada como uma resistência que o meio solo - sistema de drenagem oferece para o fluxo da água aos drenos; como $h_{s}$ é um valor tomado no início do $\Delta \mathrm{t}$ e, portanto, conhecido, a equação anterior permite o cálculo de $\eta$ e de q; a lâmina drenada é incorporada à equação de Richards através do termo $D_{r}=1 / \eta$; este termo, com dimensão $T^{-1}$, corresponde ao W(h) da Eq. 1.

\section{Verificação do modelo}

A partir das condições iniciais e de contorno já definidas, o desempenho do modelo foi avaliado comparando-se seus resultados com observações feitas na área experimental em diferentes períodos. Essas observações dizem respeito à profundidade do lençol freático no ponto médio entre dois drenos espaçados 18 m; o parâmetro representativo das condições de drenagem superficial foi admitido igual a $1 \mathrm{~mm}$.

O balanço hídrico calculado pelo modelo corresponde ao período de sua verificação e está apresentado na Tabela 2, a seguir.

Tabela 2. Balanço hídrico - Verificação do modelo SWAP

\begin{tabular}{lc}
\hline Variável & Lâmina (mm) \\
Precipitação & 383,6 \\
Drenagem Superficial & 97,1 \\
Evaporação & 120,2 \\
Drenagem Subsuperficial & 107,8 \\
Variação do armazenamento & 58,6 \\
\hline
\end{tabular}

O confronto entre valores observados e calculados é apresentado na Tabela 3.

Os valores de $\mathrm{Ef}_{1}\left(\right.$ valor ótimo = 1) e $\mathrm{Ef}_{2}($ valor ótimo = 0$)$ foram definidos pelas equações:

$$
\begin{gathered}
\mathrm{Ef}_{1}=\frac{\sum_{\mathrm{i}=1}^{\mathrm{n}}\left(\mathrm{o}_{\mathrm{i}}-\overline{\mathrm{O}}\right)^{2}-\sum_{\mathrm{i}=1}^{\mathrm{n}}\left(\mathrm{p}_{\mathrm{i}}-\mathrm{o}_{\mathrm{i}}\right)^{2}}{\sum_{\mathrm{i}=1}^{\mathrm{n}}\left(\mathrm{o}_{\mathrm{i}}-\overline{\mathrm{O}}\right)^{2}} \\
\mathrm{Ef}_{2}=\frac{\left[\sum_{\mathrm{i}=1}^{\mathrm{n}}\left(\mathrm{p}_{\mathrm{i}}-\mathrm{o}_{\mathrm{i}}\right)^{2} / \mathrm{n}\right]^{1 / 2}}{\overline{\mathrm{O}}}
\end{gathered}
$$


em que:

$$
\begin{aligned}
& \mathrm{O}_{\mathrm{i}} \text { - valor observado } \\
& \mathrm{p}_{\mathrm{i}}-\text { valor calculado } \\
& \overline{\mathrm{O}} \text { - média dos valores observados } \\
& \mathrm{n} \text { - número de valores }
\end{aligned}
$$

Tabela 3. Profundidade do lençol freático: valores observados e calculados

\begin{tabular}{ccc}
\hline Data & \multicolumn{2}{c}{ Profundidade do lençol freático (cm) } \\
\cline { 2 - 3 } $29 / 08$ & Observado & Calculado \\
$01 / 09$ & 40 & 40 \\
$02 / 09$ & 0 & 0 \\
$03 / 09$ & 10 & 11 \\
$02 / 10$ & 24 & 18 \\
$03 / 10$ & 0 & 0 \\
$04 / 10$ & 11 & 14 \\
$09 / 10$ & 25 & 20 \\
$10 / 10$ & 5 & 0 \\
$11 / 10$ & 6 & 9 \\
$12 / 10$ & 15 & 16 \\
$17 / 10$ & 29 & 22 \\
$19 / 10$ & 0 & 0 \\
$20 / 10$ & 13 & 18 \\
$21 / 10$ & 25 & 24 \\
& 30 & 25 \\
\hline
\end{tabular}

A inspeção da Tabela 3 aliada aos índices estatísticos $\mathrm{Ef}_{1}$ e $\mathrm{Ef}_{2}$, mostra ótimo desempenho do modelo na simulação de cenários que incluem diferentes condições de drenagem em solos pouco profundos.

\section{Condições adotadas nas simulações}

Critérios para mecanização: Far-se-á a avaliação das diferentes condições de drenagem com base na contabilização, dentro de cada período simulado, do número de dias úteis para atividades de mecanização. Os critérios adotados para que um dia seja considerado apto, sugeridos por van Wijk \& Feddes (1982, 1986) e Fernandes et al. (2002), são apresentados na Tabela 4.

Tabela 4. Critérios para mecanização

\begin{tabular}{lccc}
\hline Pesquisadores & $\begin{array}{c}\text { Profundidade } \\
\text { (cm) }\end{array}$ & $\begin{array}{c}\text { Potencial matricial } \\
\text { (h) (cm) }\end{array}$ & $\begin{array}{c}\text { Umidade }(\boldsymbol{\theta}) \\
\left(\mathbf{c m}^{3} \mathbf{~ c m}^{-3}\right)\end{array}$ \\
$\begin{array}{l}\text { van Wijk \& Feddes } \\
\begin{array}{l}(1982,1986) \\
\text { Fernandes et al. }\end{array}\end{array}$ & 5 & h pontual $\leq-100$ & - \\
$(2002)$ & 15 & - & $\begin{array}{r}\theta \text { médio } \leq 90 \% \leq \\
\theta(\mathrm{h}=-100)\end{array}$ \\
\hline
\end{tabular}

Cenários e períodos simulados: Os períodos simulados correspondem ao mês de setembro (06/09 a 30/09) dos anos de 1996, 97, 99 e 2000, haja vista ser este o período do ano em que se concentram as operações de preparo do solo para o cultivo de verão. As diferentes condições de drenagem foram caracterizadas pelo armazenamento máximo na superfície $\left(\mathrm{A}_{\mathrm{máx}}\right)$ que antecede o início do escoamento (drenagem superficial) e pelo espaçamento (L) e profundidade (p) dos drenos (drenagem subsuperficial). Boas condições de drena- gem superficial foram caracterizadas por $\mathrm{A}_{\text {máx }}=1 \mathrm{~mm}$, valor adotado para validação do modelo com dados de área sistematizada. Más condições de drenagem superficial foram caracterizadas por $A_{\text {máx }}=20 \mathrm{~mm}$, valor arbitrado representando áreas não sistematizadas. As condições de drenagem subsuperficial foram definidas pelos seguintes pares de valores $\mathrm{L}(\mathrm{m}) / \mathrm{p}(\mathrm{cm}):$ 5/75, 10/75, 20/75, 50/25 e 100/25. Os espaçamentos de 50 e $100 \mathrm{~m}$ e a profundidade de $25 \mathrm{~cm}$, representam a média das condições atualmente existentes nas áreas cultivadas com arroz, nos solos de várzea do Rio Grande do Sul; os demais espaçamentos e profundidade indicam cenários hipotéticos nos quais as condições de drenagem são significativamente melhoradas.

\section{RESULTADOS E DISCUSSÃO}

Apresentam-se, inicialmente, na Tabela 5, os balanços gerados pelo modelo em cada simulação, cujos resultados dizem respeito a uma boa condição de drenagem superficial.

Esses resultados mostram claramente uma relação estreita

\begin{tabular}{|c|c|c|c|c|c|}
\hline \multirow[b]{2}{*}{ Simulação } & \multicolumn{5}{|c|}{ Armazenamento máximo na superfície $=1 \mathrm{~mm}$} \\
\hline & \multirow{2}{*}{\multicolumn{2}{|c|}{$\begin{array}{r}\mathrm{L}^{1}=100 \mathrm{~m} \mathrm{~L}=50 \mathrm{~m} \\
\mathrm{P}^{2}=25 \mathrm{~cm} \mathrm{P}=25 \mathrm{~cm} \\
01 \text { set/1996 }\end{array}$}} & \multirow{2}{*}{$\begin{array}{l}\mathrm{L}=20 \mathrm{~m} \\
\mathrm{P}=75 \mathrm{~cm}\end{array}$} & \multirow{2}{*}{$\begin{array}{l}L=10 \mathrm{~m} \\
P=75 \mathrm{~cm}\end{array}$} & $\begin{array}{c}L=5 \mathrm{~m} \\
P=75 \mathrm{~cm}\end{array}$ \\
\hline & & & & & \\
\hline Precipitação (mm) & 106,3 & 106,3 & 106,3 & 106,3 & 106,3 \\
\hline $\begin{array}{l}\text { Escoamento superficial } \\
(\mathrm{mm})\end{array}$ & 56,5 & 56,3 & 53,5 & 44,7 & 18,1 \\
\hline Evaporação (mm) & 37,5 & 37,5 & 37,5 & 37,5 & 37,5 \\
\hline Drenagem (mm) & 0,1 & 0,4 & 4,7 & 16,0 & 46,2 \\
\hline $\begin{array}{l}\text { Variação do } \\
\text { armazenamento (mm) }\end{array}$ & 12,2 & 12,1 & 10,7 & 8,2 & 4,5 \\
\hline \multicolumn{6}{|c|}{02 set/1997 } \\
\hline Precipitação (mm) & 86,7 & 86,7 & 86,7 & 86,7 & 86,7 \\
\hline $\begin{array}{l}\text { Escoamento superficial } \\
(\mathrm{mm})\end{array}$ & 47,1 & 47,0 & 44,9 & 39,0 & 20,3 \\
\hline Evaporação (mm) & 43,0 & 43,0 & 43,0 & 43,0 & 43,0 \\
\hline Drenagem (mm) & 0,1 & 0,2 & 3,6 & 11,7 & 33,6 \\
\hline $\begin{array}{l}\text { Variação do } \\
\text { armazenamento (mm) }\end{array}$ & $-3,5$ & $-3,5$ & $-4,8$ & $-7,0$ & $-10,3$ \\
\hline \multicolumn{6}{|c|}{03 set/1999 } \\
\hline Precipitação (mm) & 67,7 & 67,7 & 67,7 & 67,7 & 67,7 \\
\hline $\begin{array}{l}\text { Escoamento superficial } \\
(\mathrm{mm})\end{array}$ & 29,9 & 29,7 & 26,6 & 20,5 & 10,5 \\
\hline Evaporação (mm) & 43,1 & 43,1 & 43,1 & 43,1 & 43,1 \\
\hline Drenagem (mm) & 0,1 & 0,3 & 4,7 & 13,0 & 26,4 \\
\hline $\begin{array}{l}\text { Variação do } \\
\text { armazenamento (mm) }\end{array}$ & $-5,4$ & $-5,4$ & $-6,7$ & $-8,9$ & $-12,2$ \\
\hline \multicolumn{6}{|c|}{$04 \mathrm{set} / 2000$} \\
\hline Precipitação (mm) & 160,8 & 160,8 & 160,8 & 160,8 & 160,8 \\
\hline $\begin{array}{l}\text { Escoamento superficial } \\
(\mathrm{mm})\end{array}$ & 107,5 & 106,8 & 96,8 & 72,7 & 21,2 \\
\hline Evaporação (mm) & 38,9 & 38,9 & 38,9 & 38,9 & 38,9 \\
\hline Drenagem (mm) & 0,2 & 0,9 & 10,9 & 35,0 & 87,7 \\
\hline $\begin{array}{l}\text { Variação do } \\
\text { armazenamento (mm) }\end{array}$ & 14,3 & 14,3 & 14,3 & 14,3 & 13,1 \\
\hline
\end{tabular}
entre a drenagem subsuperficial e o escoamento superficial.

Tabela 5. Balanço hídrico - simulações 01, 02, 03 e 04 - armazenamento máximo na superfície $=1 \mathrm{~mm}$

${ }^{1}$ Espaçamento entre drenos; ${ }^{2}$ Profundidade de instalação dos drenos 
A redução da lâmina drenada pelo sistema subsuperficial, decorrente do aumento do espaçamento e diminuição da profundidade dos drenos, implica em um acréscimo no escoamento superficial, de tal forma que o somatório dessas duas variáveis pouco se altera entre simulações no mesmo período. $\mathrm{O}$ aumento do espaçamento entre os drenos faz com que diminua a infiltração, de tal forma que grande parte da lâmina que deixa de ser drenada é transferida para o escoamento superficial e apenas uma pequena parcela contribui para o aumento da umidade do perfil do solo. A hipótese de boa condição de drenagem superficial (armazenamento máximo na superfície = $1 \mathrm{~mm}$ ) justifica, de certa forma, o comportamento dessas variáveis; suas relações com o espaçamento estão representadas na Figura 2A. Na Figura 2B apresenta-se unicamente, para a simulação 04 (na qual se tem a maior lâmina precipitada) o comportamento isolado das mesmas variáveis. A forma aproximadamente simétrica das curvas ratifica a transferência na mesma proporção da lâmina de uma para outra, com mais drenagem subsuperficial e menos escoamento nos menores espaçamentos.

Tomando-se como referência os perfis de umidade gerados pelo modelo, resultados mais específicos, sintetizados pelo número de dias úteis para a mecanização, são apresentados na Tabela 6.

Tabela 6. Número de dias para mecanização - simulações 01, 02, 03 e 04 - armazenamento máximo na superfície $=1 \mathrm{~mm}$

\begin{tabular}{|c|c|c|c|c|c|}
\hline & $\begin{array}{l}\mathrm{L}^{1}=100 \mathrm{~m} \\
\mathrm{P}^{2}=25 \mathrm{~cm}\end{array}$ & $\begin{array}{l}\mathrm{L}=50 \mathrm{~m} \\
\mathrm{P}=25 \mathrm{~cm}\end{array}$ & $\begin{aligned} \mathrm{L} & =20 \mathrm{~m} \\
\mathrm{P} & =75 \mathrm{~cm}\end{aligned}$ & $\begin{array}{l}L=10 \mathrm{~m} \\
P=75 \mathrm{~cm}\end{array}$ & $\begin{array}{c}\mathrm{L}=5 \mathrm{~m} \\
\mathrm{P}=75 \mathrm{~cm}\end{array}$ \\
\hline \multicolumn{6}{|c|}{ Simulação 01} \\
\hline Critério $1^{3}$ & 3 & 3 & 3 & 4 & 5 \\
\hline Critério $2^{4}$ & 3 & 3 & 3 & 5 & 6 \\
\hline \multicolumn{6}{|c|}{ Simulação 02} \\
\hline Critério 1 & 7 & 7 & 8 & 9 & 12 \\
\hline Critério 2 & 6 & 7 & 7 & 9 & 12 \\
\hline \multicolumn{6}{|c|}{ Simulação 03} \\
\hline Critério 1 & 5 & 5 & 6 & 6 & 9 \\
\hline Critério 2 & 5 & 5 & 5 & 6 & 8 \\
\hline \multicolumn{6}{|c|}{ Simulação 04} \\
\hline Critério 1 & 3 & 3 & 3 & 3 & 5 \\
\hline Critério 2 & 3 & 3 & 3 & 3 & 4 \\
\hline
\end{tabular}

1 Espaçamento entre drenos; ${ }^{2}$ Profundidade de instalação dos drenos; ${ }^{3}$ Critério de van Wijk \& Feddes (1982,1986); ${ }^{4}$ Critério de Fernandes et al. (2002)

As considerações anteriores quanto às variáveis drenagem e escoamento superficial dão suporte à diferença entre o número total de dias úteis para mecanização oferecido pelos espaçamentos de 20 e $50 \mathrm{~m}$ ou 50 e $100 \mathrm{~m}$, quando comparados com o número oferecido pelos espaçamentos de 5 e $10 \mathrm{~m}$. Os dias totalizados relacionados aos maiores espaçamentos correspondem a $72 \%$ dos dias obtidos com os menores espaçamentos; este percentual mostra que grandes diferenças entre espaçamentos não foram acompanhadas por grandes diferenças nas condições de umidade do solo; por outro lado, os valores dessas tabelas indicam que a sensibilidade do sistema à presença dos drenos se esgota nos espaçamentos de 5 e $10 \mathrm{~m}$, ou seja, as respostas são praticamente as mesmas para os demais espaçamentos. Os critérios
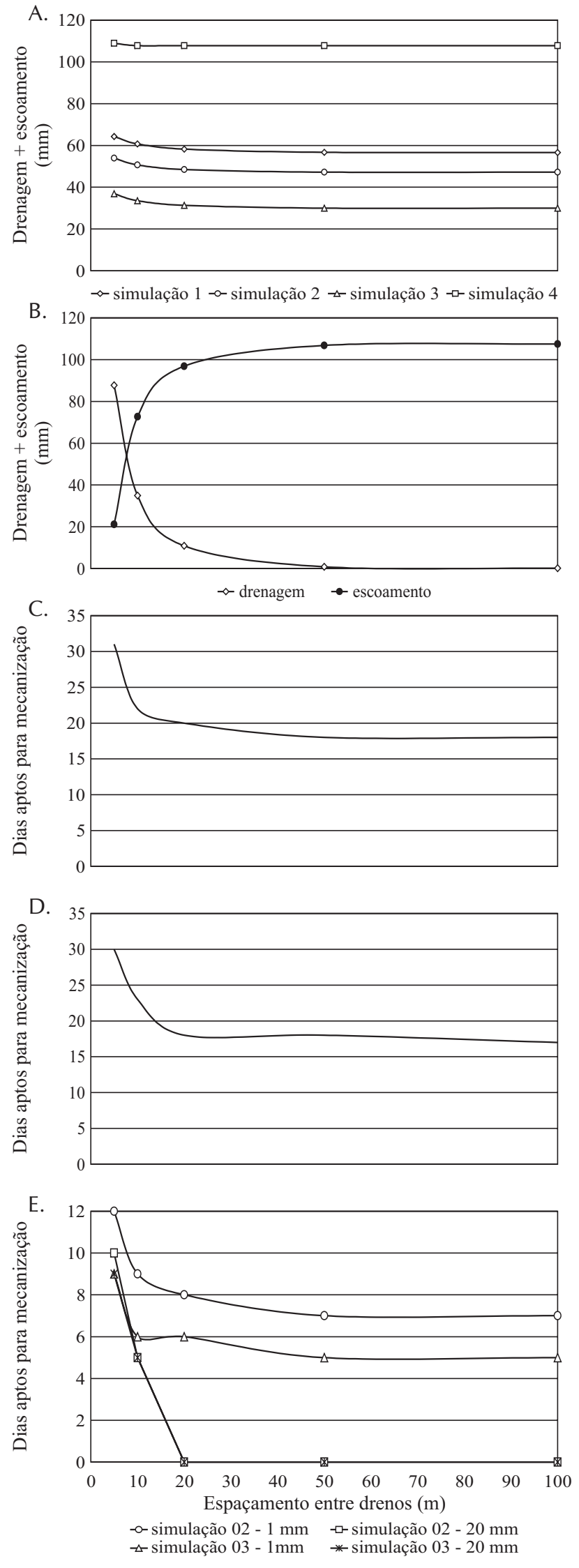

Figura 2. Drenagem + escoamento superficial vs espaçamento - simulações 01 a 04 - armazenamento máximo na superfície $=1 \mathrm{~mm}$ (A) / Espaçamento vs escoamento superficial - simulação 04 (armazenamento máximo na superfície $=1 \mathrm{~mm})(B) /$ Somatório de dias úteis para mecanização - critério 1 (C) e critério 2 (D) / Dias propícios à mecanização com diferentes condições de drenagem $(\mathrm{E})$ 
adotados e identificados nas tabelas por critério 1 e critério 2 , apontam na mesma direção já que conduzem a resultados muito próximos. Esses resultados são ilustrados na Figura 2C e Figura 2D. Na Tabela 7, novos resultados são comparados aos anteriores considerando-se que o armazenamento na superfície pode atingir até $20 \mathrm{~mm}$. Os resultados se restringem às simulações 02 e 03 e ao critério 1 . Os resultados evidenciam alterações significativas com as novas condições de drenagem superficial; essas alterações podem ser mais bem visualizadas na Figura 2E.

Tabela 7. Dias propícios à mecanização com diferentes condições de drenagem

\begin{tabular}{|c|c|c|c|c|c|c|c|c|c|c|}
\hline \multirow[b]{2}{*}{$L^{*}(m)$} & \multicolumn{5}{|c|}{$\begin{array}{l}\text { Armazenamento } \mathrm{na} \\
\text { superficie }=1 \mathrm{~mm}\end{array}$} & \multicolumn{5}{|c|}{$\begin{array}{l}\text { Armazenamento } \mathrm{na} \\
\text { superficie }=20 \mathrm{~mm}\end{array}$} \\
\hline & 5 & 10 & 20 & 50 & 100 & 5 & 10 & 20 & 50 & 100 \\
\hline Sim. 02 & 12 & 9 & 8 & 7 & 7 & 10 & 5 & 0 & 0 & 0 \\
\hline Sim. 03 & 9 & 6 & 6 & 5 & 5 & 9 & 5 & 0 & 0 & 0 \\
\hline
\end{tabular}

* espaçamento entre drenos

Novamente, o comportamento das variáveis drenagem e escoamento superficial pode ser utilizado para interpretação dos resultados. No caso dos menores espaçamentos (5 e $10 \mathrm{~m}$ ) o decréscimo na drenagem superficial é, em grande parte, transferido para a drenagem subsuperficial, o que faz com que as reduções no número de dias para mecanização sejam de 28,6\% (simulação 03) e 6,7\% (simulação 04); já para os demais espaçamentos isto não acontece e a lâmina, que dei-

A.
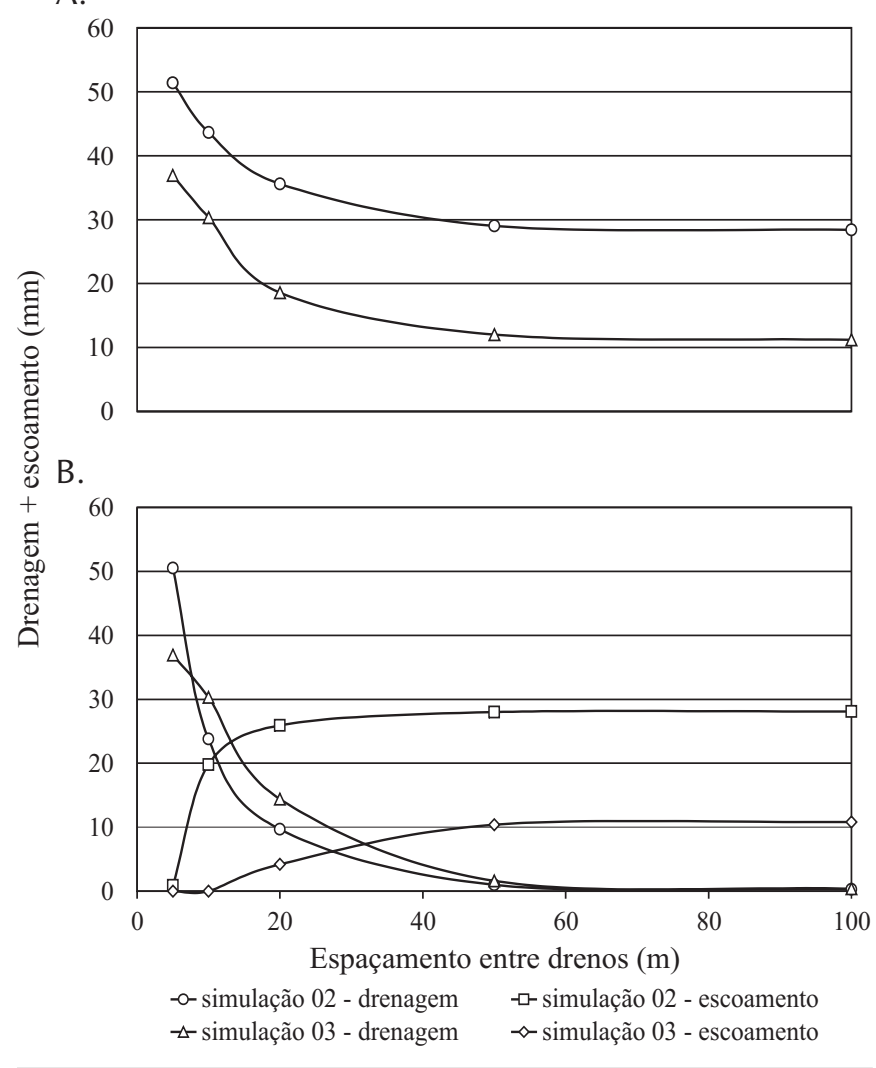

Figura 3. Simulações 02 e 03 - armazenamento máximo na superfície = $20 \mathrm{~mm}$ : Drenagem + escoamento superficial vs espaçamento (A) e espaçamento vs drenagem vs escoamento superficial (B) xa de ser drenada pela superfície, passa a contribuir, de forma efetiva, para a elevação da umidade do solo; então, a redução no número de dias para mecanização atinge $100 \%$ nas duas simulações; o comportamento dessas duas variáveis somadas é ilustrado na Figura 3A.

Tem-se, na Figura 3A, um comportamento distinto do que se verifica na Figura 2A, em que as mesmas variáveis são relacionadas. A não horizontalidade de suas linhas indica, no caso dos maiores espaçamentos, que só uma pequena parcela da lâmina deixa de escoar pela superfície e se transfere para a que é drenada pelo sistema subsuperficial. O comportamento isolado dessas variáveis é representado na Figura 3B. A não-simetria das curvas em relação ao eixo vertical ratifica as observações anteriores, ao mostrar que a transferência entre as variáveis não é proporcional, ou seja, o crescimento que se verifica em uma não é acompanhado por igual decréscimo na outra.

\section{CONCLUSÕES}

1. A aderência entre valores observados e calculados justificou plenamente o emprego do modelo para simulação do comportamento das variáveis que definem a dinâmica da drenagem em solos pouco profundos.

2. As simulações que relacionaram drenagem e mecanização, mostraram que as formas convencionais de drenagem superficial e subsuperficial não podem ser avaliadas de forma isolada.

3. Quando as condições de escoamento superficial foram admitidas como boas (armazenamento máximo $=1 \mathrm{~mm}$ ), os perfis de umidade se mostraram, em alguns casos, compatíveis com a mecanização, mesmo para grandes espaçamentos entre drenos subsuperficiais.

4. Só se verificou alteração significativa nesses perfis quando o espaçamento entre os drenos subsuperficiais foi bastante reduzido.

5. Para más condições de drenagem na superfície (armazenamento máximo $=20 \mathrm{~mm}$ ), os perfis de umidade só se mostraram adequados quando os drenos subsuperficiais foram dispostos muito próximos.

6. Verifica-se claramente que o incremento no número dias aptos para mecanização pode ser atingido com boas condições de drenagem superficial.

\section{LITERATURA CITADA}

Agrianual. Anuário Estatístico. Rio de Janeiro: FNP Consultoria \& Comércio. 1999. 956 p.

Celia, M. A.; Bouloutas, E. T.; Zarba, L. Z. A general mass-conservative numerical solution for the unsaturated flow equation. Water Resources Research, v.26, n.7, p.1483-1496. 1990.

EMBRAPA-Empresa Brasileira de Pesquisa Agropecuária. Centro Nacional de Pesquisa de Solos. Ministério da Agricultura e do Abastecimento. Sistema Brasileiro de Classificação de Solos. Brasília. 1999. 412p. 
Fernandes, H. C.; Hamakawa, P. J.; Lanças, K. P. Metodologia e cálculo dos dias trabalháveis com máquinas florestais na região de Botucatu, SP. Engenharia Agrícola, Jaboticabal, v.22, n.1, p.68-74. 2002.

Grando, M. Z., coord. Agropecuária do Rio Grande do Sul 1980 - 1985: A caminho da eficiência. Porto Alegre: Fundação de Economia e Estatística. Siegfried Emanuel Heuser. 1996. 160p.

Kool, J. B.; Parker, J. C. Development and evaluation of closed form expressions for hysteretic soil hydraulic properties. Water Resources Research, v.23, n.1, p.105-14. 1987.

Kroes, J. C.; van Dam, J. C.; Huygen, J.; Vervoort, R. W. User’s guide of SWAP version 2.0. Wageningen: Wageningen Agricultural University/DLO. 1998. 105p.

Matzenauer, R.; Bergamaschi, H.; Berlato, M. A. Consumo de água e disponibilidade hídrica para para milho e soja, no Rio Grande do Sul. Porto Alegre: FEPAGRO, 2002. 105p.

Mualem, Y. A new model for predicting the hydraulic conductivity of unsaturated porous media. Water Resources Research. v.12, n.3, p.513-522. 1976.

van Dam, J. C. Field-scale water flow and solute transport : SWAP model concepts, parameter estimation and case studies. Wageningen: Wageningen Agricultural University. 2000. 167p. PhD Thesis van Dam, J. C.; Huygen, J.; Wesseling, J. G.; Feddes, R. A.; Kabat, P., Walsum, P. E. V.; Groenendijk, P.; van Diepen, C. A. Theory of SWAP version 2.0 ; simulation of water flow, solute transport and plant growth in the soil - water - atmosphere - plant environment. Wageningen: Wageningen Agricultural University/DLO. 1997. 167p.

van Genuchten, M. Th. A closed form equation for predicting the hydraulic conductivity of unsaturated soils. Soil Science Society American Journal, v.44, p.892-898. 1980.

van Wijk, A. L. M.; Feddes, R. A. A model approach to the evaluation of drainage effects. In: Land Drainage Seminar, 1982, Cambridge. Proceedings... Cambridge: Dublin: Kinsealy research Centre. 1982. p.131-149.

van Wijk, A. L. M.; Feddes, R. A. Simulating effects of soil type and drainage on arable crop yield. In: Agricultural Water Management Symposium, 1986, Arnhem. Proceedings... Arnhem: Wageningen: Institute for Land and Water Management Research. 1986. p.97-112.

van der Molen, W. H.; Wesseling, J. A solution in closed form and a series solution to replace the tables for the thickness of the equivalent layer in Hooghoudt's drain spacing formula. Agricultural Water Management, Amsterdam, v.19, p.1-16. 1991. 
